\title{
Reconstruction of palate with buccal fat pad secondary to resection of desmoplastic ameloblastoma
}

\section{Bhimappa Mallappa Rudagi, Manjunatha Reddy Bandral, Reshma Hammannawar, Pritish Harish Padgavankar}

Department of Oral and Maxillofacial Surgery, A.C.P.M. Dental College, Dhule 424001, Maharastra, India.

Address for correspondence: Dr. Pritish Harish Padgavankar, Department of Oral and Maxillofacial Surgery, A.C.P.M. Dental College, Sakri Road, Dhule 424001, Maharastra, India. E-mail: pritishharishchandra@gmail.com

\begin{abstract}
Desmoplastic ameloblastoma (DA) is an unusual variant of ameloblastoma exhibiting important differences in the anatomical distribution, radiographic features and histologic appearance compared with the classic type of ameloblastoma. The purpose of this paper is to report a case of DA in the anterior left maxilla and to describe a simple method of reconstruction with the use of buccal fat pad (BFP). BFP is an excellent choice for reconstruction of small to medium sized defects. It should be manipulated gently and hemostasis should be achieved meticulously during this surgery. It should not be sutured under tension.
\end{abstract}

Key words:

Ameloblastoma, buccal fat pad, desmoplastic ameloblastoma, reconstruction, resection

\section{INTRODUCTION}

Ameloblastoma is a rare, benign, slowly growing and locally invasive neoplasm that accounts for about $1 \%$ of all cysts and tumors of the jaws and $18 \%$ of the various odontogenic neoplasms. ${ }^{[1]}$ In the recent histological classification of odontogenic tumors from the World Health Organization (WHO), ameloblastoma is defined as a benign, locally invasive epithelial odontogenic neoplasm of putative enamel organ origin. ${ }^{[2]}$ Various clinical types of ameloblastoma are solid-multicystic, unicystic, malignant and rare peripheral type. Histopathologically classified as follicular, acanthomatous, granular, basal, desmoplastic and plexiform ameloblastoma. ${ }^{[3]}$ Desmoplastic ameloblastoma (DA) was first described in detail by Eversole et al. ${ }^{[4]}$ In 1984 and in recent WHO classification of odontogenic tumors, it is defined as

\begin{tabular}{|l|l|}
\multicolumn{2}{|c|}{ Access this article online } \\
\hline Quick Response Code: & Website: \\
\hline & www.parjournal.net \\
\cline { 2 - 3 } & \\
\hline
\end{tabular}

"a variant of ameloblastoma with specific clinical, imaging and histological features". ${ }^{[5]}$ Follicular and plexiform are the commonly encountered variants accounting for $32.5 \%$ and $28.2 \%$ respectively, followed by the acanthomatous subtype with $12.1 \%$ while desmoplastic is extremely uncommon with incidence rates ranging from $4-13 \%$ in

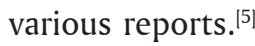

According to Shafer, ${ }^{[6]}$ the follicular type of ameloblastom had the highest rate of recurrence (29.5\%), plexiform subtype showed a $16.7 \%$ recurrence rate and acathomatous type of ameloblastoma showed only $4.5 \%$ recurrence rate. DA requires special attention as it exhibits important differences in the anatomical distribution, radiographic and histomorphological compared with the classic type of ameloblastoma. ${ }^{[2]}$

The purpose of this article is to present a case of DA in the anterior left maxilla and to describe a simple method of reconstruction with the use of buccal fat pad (BFP).

\section{CASE REPORT}

A 25-year-old male patient reported to the Department of Oral and Maxillofacial Surgery, A.C.P.M. Dental College, Dhule, Maharastra, India with the complaint of swelling in the left anterior region of the upper jaw since 3 months. 
He revealed a history of insidious onset as a small nodule, gradually reaching the present size. The patient had no history of trauma to this region. Extraoral examination showed facial swelling with obliteration of left nasolabial fold. Swelling was oval, smooth, approximately $3 \mathrm{~cm} \times 3 \mathrm{~cm}$ in size, extending antero-posteriorly from left ala of nose to canine fossa and superoinferiorly from left infraorbital foramen region to the left corner of upper lip. The skin over the swelling appeared normal without pain, pus discharge, or paresthesia. No lymphadenopathy was noted.

Intraorally, obliteration of upper buccal vestibule was seen on the left side [Figure 1]. The swelling extended from the upper left lateral incisor to the left second premolar buccally and palataly, superoinferiorly from the vestibular mucosa to the marginal gingiva of the teeth on buccal side and on the palatal side about $2 \mathrm{~cm}$ from the midline to marginal gingiva. The swelling was approximately $2.5 \mathrm{~cm} \times 3 \mathrm{~cm}$ in size, well defined with intact mucosa causing bicortical expansion. On palpation, the swelling was nontender, bony hard, nonfluctuant, noncompressible, nonreducible and nonpulsatile, fixed to the underlying structure. No dental abnormality was seen in the region. An orthopantogram of the region revealed a triangular radiolucency with ill-defined margins, causing mesial displacement of root of left lateral incisor and canine and proximity with distal displacement of root of the first and second premolar. Water's view showed haziness over the left maxillary sinus [Figure 2].

The overall clinical features were suggestive of an odontogenic tumor, probably an ameloblastoma, with differential diagnosis of a cyst, abscess, canine space infection, monostotic fibrous dysplasia, adenomatoid odontogenic tumor, central or peripheral giant cell granuloma, aneurysmal bone cyst. The radiographical differential diagnosis includes unilocular ameloblastoma, tumor of maxillary sinus, odontogenic keratocyst, radicular cyst, adenomatoid odontogenic tumor, ossifying fibroma, and odontogenic myxoma. To confirm the diagnosis, fine needle aspiration cytology was performed, but it was inconclusive, so incisional biopsy was performed, and histopathologic evaluation diagnosis of DA was established [Figure 3].

After confirmation of diagnosis as DA from the clinical, histological, and radiological examination, planning for surgical resection and reconstruction was done. Under general anesthesia and using a maxillary vestibular approach, incision was placed in the left buccal vestibule extending from the left central incisor to left second molar region, exposure of the lesion was done. Extraction of left upper central incisor and left second molar was done and osteotomy cut was performed on the buccal side from the extraction socket and connected to each other with a horizontal osteotomy cut just beneath the infraorbital foramen protecting infraorbital neurovascular bundle. On palatal side, incision was given from the socket of left upper central incisor to left second molar with an electrocautery, and a vertical osteotomy cut was performed. Partial maxillectomy with



Figure 1: Intraorally, swelling of $3 \mathrm{~cm} \times 3 \mathrm{~cm}$ in size in the left anterior maxilla with intact mucosa causing bicortical expansion

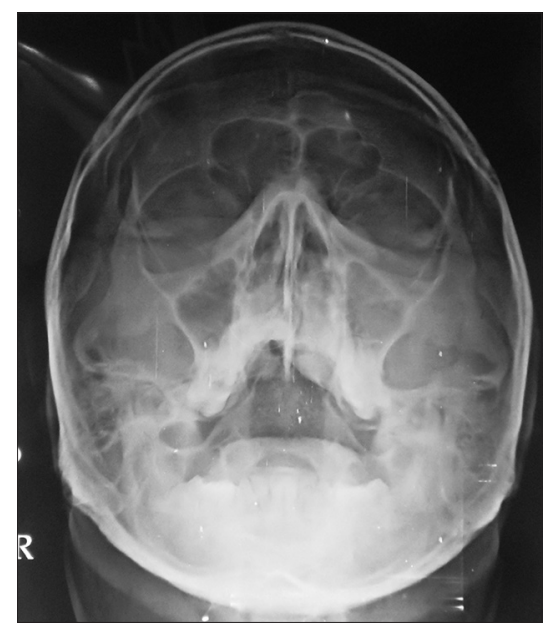

Figure 2: Water's view showed haziness over the left maxillary sinus

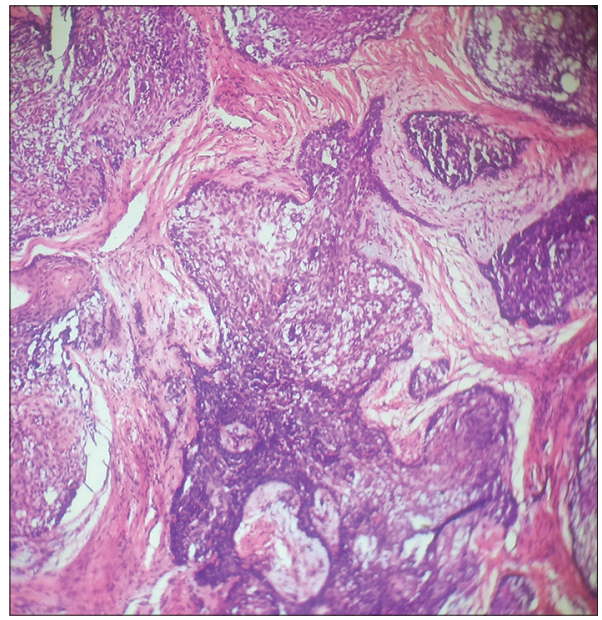

Figure 3: $\mathrm{H}$ and E stained section showed irregularly proliferating tumor island surrounded by dense fibrous stroma and extensive desmoplasia compressing the odontogenic epithelial island from the periphery

resection of the complete lesion $(4 \mathrm{~cm} \times 4 \mathrm{~cm})$ was done [Figure 4]. After resection of the lesion, margins were examined, and immediate reconstruction with BFP was planned. BFP was harvested by exposing the left buccal mucosa and bluntly dissecting the area until BFP was visible and nontoothed forcep was used to grasp the BFP. It was gently teased and pulled to the wound. 
The BFP was expanded and sutured with 4-0 vicryl to the underlying wound. A saframycin based oral pack was secured over the palate with 3-0 vicryl suture for primary protection for 1 week till the epithelization begins, and strict soft diet was followed [Figure 5]. The patient was kept on periodic recall every 2 months during the 1 year follow-up [Figure 6].

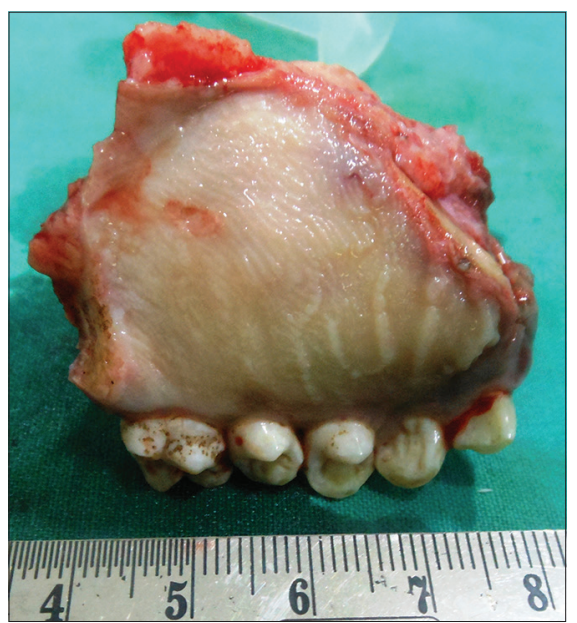

Figure 4: Partial maxillectomy was carried out and resection of the complete lesion was done

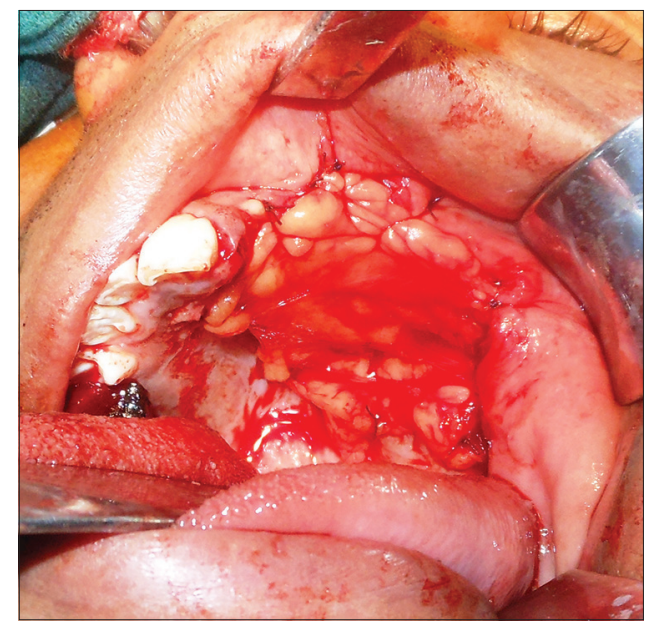

Figure 5: Reconstruction with buccal pad of fat. Immediate intra-operative picture

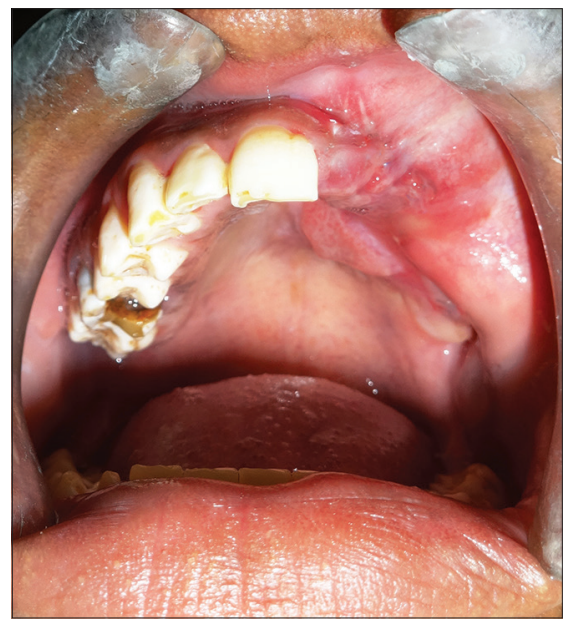

Figure 6: Postoperative picture after 1 year

\section{DISCUSSION}

The use of BFP as a grafting source was first described in 1977 by Egyedi. ${ }^{[7]}$ Anatomically BFP is a fatty mass in the buccal space of the cheek. It comprises 3 lobes: anterior, intermediate and posterior with 4 extensions, that is, buccal, pterygoid, pterygopalatine and temporal (superficial and profound). It is fixated by ligaments to the maxilla, posterior zygomatic bone, inner and outer rim of the orbital fissure, temporalis tendon and buccinators membrane. ${ }^{[8]}$ It is intimately associated with muscles of mastication, facial nerve and parotid duct. The use of the BFP as a grafting source in closure of intra-oral defects has gained popularity, because of the ease of harvesting, simplicity, versatility, rich blood supply, low complication rate and quick surgical techniques. ${ }^{[5]}$ Tideman et al. $^{[9]}$ showed that the BFP is epithelialized within 3-4 weeks and therefore further skin graft are not required [Figure 5]. According to Alkan et al., ${ }^{[10]}$ the success rate of the use of BFP is relatively high in all comparative studies.

In our case, we performed a partial maxillectomy procedure and complete resection of the lesion was done, after which a postsurgical defect was present in left anterior maxillary region. An immediate reconstruction with BFP was planned, and BFP was harvested from the left buccal mucosa. Intra-oral postsurgical defects reconstruction is always challenging one due to anatomical constraints and the specialized nature of intra oral tissues. The principal arterial supply to BFP is from buccal and deep branches of maxillary artery, from transverse facial branch of superficial temporal artery and from few branches of the facial artery. ${ }^{[1]]}$ There should remain a reasonable size pedicle attached to the BFP to provide it with the crucial blood supply in the 1 st week of its life. It is essential to stop bleeding from BFP during surgery with the help of electrocautery or small ligatures as a failure to do so leads to the formation of buccal haematoma and could compromise the viability of the flap. It is also important to be meticulous in dissecting out the flap, protecting the small branches of the facial nerve and parotid duct.

Buccal fat pad is morphologically different from subcutaneous fat but similar to orbital fat. The mean volume of BFP is about $10 \mathrm{~mL}$ and its mean thickness is $6 \mathrm{~mm}$ while the approximate weight is of $9.3 \mathrm{~g}$. It can successfully be used for covering small to medium defects of about $4 \mathrm{~cm}$ in diameter. ${ }^{[12]}$ BFP has also been employed in the closure of surgical defects following tumor excision, excision of leukoplakia and submucous fibrosis, as well as closure of primary and secondary palatal clefts. ${ }^{[13]}$ Flap should be sutured gently to the borders of the defects and ideally there should not be any stretch within the tissue. Overstretching the tissue can lead to fragmentation of the flap and in the long term can lead to ischemic necrosis at the edges. Disadvantage with the use of the BFP flap is hematoma formation, partial necrosis, excessive scarring, infection or facial nerve injury. None of these changes was noted in our case. The use of BFP in patients with 
prior local radiotherapy, malar hypoplasia, thin cheeks or Down's syndrome is contraindicated. ${ }^{[14]}$

Approximately half of the desmoplastic lesions are located in the maxilla, and the vast majority of them occur in the anterior or premolar portion of the jaws. Maxillary lesions are more insidious than mandibular tumors owing to the proximity of vital structures and the maxillary sinus. ${ }^{15]}$ Studies verify that DA shows a tendency to recur and the rate of recurrence is reported within the range of other histological subtypes. ${ }^{[1,6]}$ Since DA tends to infiltrate the surround bone trabeculae, curettage is considered an inappropriate treatment for DA. Therefore, block excision is the most widely accepted form of treatment for such lesions of the maxilla, particularly because recurrence is almost inevitable and difficult to treat. ${ }^{[11,16]}$ The majority of DA cases reported in the literature have been treated by resection, most likely due to ill-defined borders and an aggressive biological behavior. ${ }^{[17]}$

Various surgical techniques have been suggested for the closure of oral defects such as primary closure, split-thickness skin graft, allogenic graft, with local flaps such as buccal advancement flap, palatal pedicled flap or double layered closure flaps using buccal and palatal tissues. However, the aforementioned procedures produce large denuded areas, result in decrease of vestibular sulcus and cannot be used to close large defects. Regional flaps including tongue flap, temporalis muscle or nasolabial flaps, etc., have also been successfully used for intra-oral reconstruction but generally preferred for defects of larger dimensions. ${ }^{[14,18]}$

In conclusion, BFP is one of the reliable methods which can be used for the replacement and reconstruction of the oral mucosa due to postsurgical defect. BFP is an excellent choice for reconstruction of small to medium size defects. It should be manipulated gently and hemostasis should be achieved meticulously during this surgery. It should not be sutured under tension. Easy mobilization of the BFP and its excellent blood supply leading to rapid healing of wound and minimal donor site morbidity makes it a method of choice.

\section{REFERENCES}

I. Reddy NV, Issrani R, Reddy S, Prabhu N, Nigam NK. Desmoplastic ameloblastoma-a case report with literature review. J Res Adv Dent 20 I3;2:95-100.

2. Reichart PA, Philipsen HP, Sonner S. Ameloblastoma: biological profile of 3677 cases. Eur J Cancer B Oral Oncol 1995;3। B: 86-99.

3. Rastogi V, Pandilwar PK, Maitra S. Ameloblastoma: an evidence based study. J Maxillofac Oral Surg 2010;9:173-7.

4. Eversole LR, Leider AS, Hansen LS. Ameloblastomas with pronounced desmoplasia. J Oral Maxillofac Surg 1984;42:735-40.

5. Angadi PV, Kale A, Hallikerimath S, Kotrashetti V, Mane D, Bhatt P, Shukla D.'Hybrid' desmoplastic ameloblastoma: an unusual case report with immunohistochemical investigation for TGF- $\beta$ and review of literature. East J Med 2011;16:9-17.

6. Rajendran R. Cysts and tumors of odontogenic origin. In: Rajendran R, Sivapathasundharam B, editors. Shafer's Textbook of Oral Pathology. 5th ed. Noida: Reed-Elsevier India Private Limited; 2006. p. 357-432.

7. Egyedi P. Utilization of the buccal fat pad for closure of oro-antral and/or oro-nasal communications. J Maxillofac Surg 1977;5:24I-4.

8. Durrani Z. Buccal fat pad flap in reconstruction of oral cavity defects: a case series of five patients. JKCD 20I2;2:83-5.

9. Tideman $\mathrm{H}$, Bosanquet $\mathrm{A}$, Scott J. Use of the buccal fat pad as a pedicled graft. J Oral Maxillofac Surg 1986;44:435-40.

10. Alkan A, Dolanmaz D, Uzun E, Erdem E. The reconstruction of oral defects with buccal fat pad. Swiss Med Wkly 2003; I33:465-70.

II. Laxmana AR, Gogineni SB, Thomas PS, Shetty SR. Desmoplastic ameloblastoma-a report of two clinical cases. Braz J Oral Sci 2010;9:137-4I.

12. Chakrabarti J,Tekriwal R, Ganguli A, Ghosh S, Mishra PK. Pedicled buccal fat pad flap for intraoral malignant defects: a series of 29 cases. Indian J Plast Surg 2009;42:36-42.

13. Ahmad SA, Khan S, Ahmad MS. Buccal fat pad reconstruction of oral mucosa in leukoplakia. Ozean J Med Sci 20I0;1:13-8.

14. Mohan MC, Manimaran K. Reconstruction of intra oral post surgical defects by buccal pad of fat-A clinical study. I Indian Acad Dent Spec 2010;1:1-4.

I5. Yazdi I,Seyedmajidi M, Foroughi R. Desmoplastic ameloblastoma (a hybrid variant): report of a case and review of the literature. Arch Iran Med 2009; I2:304-8.

16. dos Santos EP, Araújo FE, Valido DP, Lima SO, de Albuquerque RL Jr, Soares AF. Desmoplastic ameloblastoma mimicking a periapical lesion. Rev Odontol Ciênc 20I0;25:306-9.

17. Amaral MB, Freire-Maia B, Serpa MR, Mesquita RA. A case report of desmoplastic ameloblastoma. J Clin Exp Dent 2010;2:149-52.

18. Grace $S$, Madhulaxmi. The use of buccal fat pad reconstruction in oral submucous fibrosis.J Med Sci Clin Res 2014;2:549-54.

How to cite this article: Rudagi BM, Bandral MR, Hammannawar R, Padgavankar PH. Reconstruction of palate with buccal fat pad secondary to resection of desmoplastic ameloblastoma. Plast Aesthet Res 2015;2:91-4.

Source of Support: Nil, Conflict of Interest: None declared.

Received: 09-09-2014; Accepted: 14-10-2014 